\title{
Intermediarios de información dentro del modelo del gobierno abierto
}

\author{
Information intermediaries within the open government model
}

\author{
Ricardo Villegas Tovar (1), Juan Carlos Marcos Recio (2)
}

(1) Instituto de Ciencias de Gobierno y Desarrollo Estratégico, Benemérita Universidad Autónoma de Puebla. Calle 4 Sur No. 104, Puebla, México. 72000; ricardo.villegas@correo.buap.mx (2) Sección Departamental de Biblioteconomía y Documentación, Facultad de Ciencias de la Información UCM. Av. Ciudad Universitaria s/n. 28040; jmarcos@ucm.es

\begin{abstract}
Resumen
A partir de una breve revisión de las más recientes etapas de evolución de la administración pública, y enfatizando el rol que la gestión de la información ha tenido en ellas, se presenta el modelo del gobierno abierto como una herramienta para conformar una sociedad objetivamente informada y participativa. Para ello, se exponen los componentes del gobierno abierto, que encuentran en los datos abiertos su principal aliado para transparentar el actuar del gobierno y hacer partícipe a la ciudadanía en la toma de las decisiones. Se concluye ubicando la oportunidad que representa contar con agentes intermediarios (incluidos los bibliotecarios) que acerquen los contenidos de los datos abiertos al común de la población a partir del desarrollo de plataformas y habilidades englobadas en el concepto de data-literacy.
\end{abstract}

Palabras clave: Gobierno abierto. Datos abiertos gubernamentales. Ecosistemas. Intermediarios de información. Infomediarios.

\section{Introducción}

La información, entendida como el producto de la recopilación e interpretación de datos para la toma de decisiones, ha sido un elemento particularmente apreciado a lo largo de las diferentes etapas evolutivas de la administración de los bienes públicos. Como ejemplo de ello se ubica en los primeros años del siglo pasado a las teorías propuestas por el economista Max Weber. El al plantear un modelo de organización de la administración pública basado en el establecimiento de puestos gerenciales y mecanismos de control de procedimientos, auguraba el cumplimiento de objetivos. Bajo este parámetro de trabajo la información se convirtió en el activo derivado del monitoreo de tales procedimientos. Por tanto, en la medida que se cumplieran estándares de desempeño sería posible demostrar la eficacia y legalidad en el actuar del gobierno. Habiéndose instaurado una serie de esquemas de auditoría, la administración que racionalizaba el uso de los recursos sería una consecuencia favorable, sin embargo, la práctica evidenció un resultado contrario. La concentración de facultades en gerentes que se

\begin{abstract}
Based on a brief review of the most recent evolution stages of the public management, emphasizing the role that information management has had in them, the open government model is introduced as a tool of the collaborative government to form an objectively informed society. With this aim, the pillars of the open government model are explained, finding open government data as its main ally to make government's actions transparent and involve citizenship in the decision-making process. We conclude locating intermediary agents (including librarians) as bridging agents joining open government data and population through the development of platforms and skills included in the data-literacy concept.
\end{abstract}

Keywords: Open government. Open government data. Ecosystem. Information intermediaries. Infomediaries.

ocupaban más en supervisar el desempeño de su personal que en proveer servicios generó una falta de atención de las necesidades sociales. A este hecho su sumó la asignación desafortunada de prebendas por parte de algunos de esos gerentes a favor de grupos selectos.

La consecuencia de estos actos fue la necesaria creación de instituciones de contrapeso tales como organismos fiscalizadores y leyes de acceso a la información pública que sirvieran para transparentar el manejo de los bienes públicos y sancionar los abusos. Así, desde finales de la década de los 60 s tuvieron origen diferentes leyes en materia de acceso a la información, como lo fue el Freedom of Information Act de los EE. UU. o su equivalente en Reino Unido.

Llegadas las décadas de los 80 s y 90 s la administración pública vivió una etapa renovada bajo los cambios enmarcados en el concepto de la Nueva Gestión Pública. En este modelo la información nuevamente ocupó un lugar preponderante como mecanismo de verificación del cumplimiento de las tareas de la administración pública. Si bien persistieron los mecanismos de control, éstos ya no 
se enfocaban únicamente en la verificación del cumplimiento de los procedimientos administrativos, sino también en la búsqueda de mecanismos para ofrecer soluciones tendientes a la generación del bienestar de la sociedad. Con la llegada de nuevas tecnologías para la gestión de la información también aparecieron nuevas opciones para la atención de las necesidades de la ciudadanía. Conceptos como el de transparencia y participación ciudadana tuvieron mayor presencia dentro de la administración pública debido al papel preponderante que la información jugaba en el cumplimiento de procesos, planes y resultados del gobierno (García, 2007).

\subsection{De la gobernanza al gobierno abierto}

Más allá del ejercicio del derecho de acceso a la información pública, y a pesar de la legitimidad in crescendo que ha venido representando la concreción del modelo de democracia directa para la elección de representantes, la incredulidad por parte de la ciudadanía igualmente ha ido acrecentándose en la medida en que se cuestiona sobre las capacidades del gobierno para resolver por sí solo todos los problemas sociales. Hoy más que cuestionarse la legitimidad del puesto del representante, se cuestiona su capacidad para gobernar. Todo esto se da en un contexto particular, en el de la sociedad de la información que se caracteriza por contar con vastos contenidos informacionales, con dispositivos electrónicos asequibles para consultarlos, con redes de conexión y habilidades intelectuales para transformar tales contenidos en conocimiento. Por tanto, al igual que sucedió con el cambio de la web 1.0 a la 2.0, la sociedad con todas estas herramientas empieza a dejar de ser un mero espectador de las actividades del gobierno y se perfila como un actor activo que participa objetivamente en la toma de las decisiones que redunden en un beneficio colectivo. Para alcanzar tal estadio, el factor informacional nuevamente se asume como un puente de múltiples carriles por el que transitan continuamente los reportes del actuar del gobierno, los requerimientos de la sociedad, las acciones colaborativas generadas entre el gobierno y la ciudadanía, las propuestas que emanan estrictamente de la ciudadanía, etc.

Bajo estos antecedentes se encuentra como una oportunidad que gobierno y sociedad compartan datos, información, conocimientos y habilidades para atender de mejor manera las necesidades de todos. Esto es lo que ha sido definido como gobernanza, como la colaboración del mayor número posible de actores en los procesos que tienen que ver con el diseño, implementación, coordinación, monitoreo, regulación y evaluación de políticas públicas (Pierre, 2000).
Con tal perspectiva es visible cómo gobierno y sociedad se convierten indistintamente en productores y consumidores de grandes cantidades de datos y de información. Esta base de contenidos al ser gestionada de manera adecuada se convertiría en el sustento del diálogo que permitiría concretar las funciones del gobierno: la definición del rumbo hacia dónde debe moverse la sociedad, la definición de los valores sociales, los medios para alcanzarlos y el papel que jugarán los actores sociales en el proceso (Aguilar, 2007).

Para que esta propuesta prospere es necesaria la intervención de instituciones y medios que articulen las aportaciones de unos y otros actores. De entre ellas, sobresale el modelo del Gobierno Abierto, que comparte elementos del movimiento open y en consecuencia promueve la libertad de acceso a contenidos informacionales con el fin de permitir su reutilización. En este sentido, el gobierno abierto se presenta como una herramienta de la gobernanza que busca fortalecer el modelo mismo de democracia a partir de hacer más partícipe a la ciudadanía en el diseño, implementación, evaluación y mejora de los servicios públicos. Para lograr esos objetivos se sustenta en tres grandes pilares: transparencia, colaboración y participación ciudadana (Naser \& Ramírez, 2014).

Por tanto, la transparencia podría entenderse como el resultado del ordenamiento y puesta a disposición del ciudadano de toda aquella información de carácter público que al ser consultada permita la construcción de argumentos objetivos para cuestionar, y mejor aún, proponer mejoras. Para ello, el establecimiento de un andamiaje legal que instruya el ordenamiento y los medios de consulta de la información pública se presentan como el antecedente directo del concepto mismo de transparencia. Para medir el grado de consolidación de este concepto Gascó (2014) plantea que al menos debería calificarse la existencia, calidad y grado de implementación de las leyes en materia de acceso a la información pública, así como la disponibilidad de portales de transparencia que incluyan indicadores de desempeño y, finalmente, la disponibilidad de portales que permitan la descarga y reutilización de conjuntos de datos (data-sets) que reporten el actuar del gobierno.

En cuanto a los conceptos de participación y colaboración la propia Gascó nos invita a evitar la confusión al parecer que estos son sinónimos. Mientras que la participación se refiere al involucramiento de la ciudadanía en los procesos de deliberación pública (votaciones, referéndums, etc.), la colaboración lleva a los ciudadanos al proceso mismo de elaboración de la política a partir de abrirles la puerta del cabildo para el planteamiento de iniciativas de leyes, políticas públicas, diseño de servicios, etc. (Gascó, 2014). Para acentuar la 
importancia del trabajo colaborativo entre gobierno y ciudadanía para la atención de los problemas sociales, la Organización para la Cooperación y el Desarrollo Económico propone una definición de gobierno abierto al referirse a él como una cultura de la gobernanza basada en políticas públicas y prácticas innovativas y sustentables inspiradas por los principios de transparencia, responsabilidad y participación que fomente la democracia y el crecimiento inclusivo (OECD, 2017).

De la lectura de estas líneas se puede inferir que la apuesta última del modelo del gobierno abierto se dirige hacia la creación de condiciones favorables para el desarrollo social. Por tanto, si la cadena de valor del gobierno abierto encuentra su punto central en la información, entonces es necesario identificar cuáles son las fuentes de tal información. El actuar cotidiano de los funcionarios del gobierno comúnmente deriva en registros de trámites, en reportes, en censos y en un sinfín de documentos con elementos cualitativos y cuantitativos. Tales elementos pueden ser extraidos y compilados con la asistencia de procedimientos computacionales, generando con ello bases de datos que al ser analizadas por medios estadísticos permitan la identificación de patrones y en consecuencia, el hallazgo de nueva información. Por tanto la extracción de tales contenidos da pie a la identificación de los denominados datos de gobierno, que al estar organizados permitan su libre consulta y reutilización, convirtiéndose así en la base de operación de la transparencia, la colaboración y la participación que promueve el modelo del gobierno abierto.

\subsection{El ecosistema del gobierno abierto}

Bajo la metáfora que permite la aplicación de la palabra ecosistema para referirse a la identificación de elementos que se interrelacionan bajo determinados intereses, se hace oportuno analizar el ecosistema del gobierno abierto. De esta forma se facilita ubicar quiénes son los involucrados en esta dinámica, cómo provocan interdependencias e inclusive cómo pueden generar valores agregados. Para lograr tal objetivo se hace indispensable identificar el contexto del tiempo y espacio que enmarca la interacción de los actores. Para ello Harrison et al. (2014) propone que los marcos de referencia a analizar sean los contextos legales, políticos y económicos. Como es previsible el legal hace referencia a la existencia de normativas que instruyan a la recolección, organización y acceso a la información que es generada por el gobierno, e inclusive la que se produce por la avenencia continua de la ciudadanía con su gobierno. Así, el ejercicio del derecho de acceso a la información pública no sólo se respalda por la apertura de los contenidos, sino por la existencia de mecanismos judiciales que aseguren la consulta expedita para los fines que mejor convengan al ciudadano.

En el contexto político pueden ubicarse al menos dos momentos que impulsaron la implementación del modelo del gobierno abierto, el primero, la iniciativa del presidente Obama quien en la renovación de su mandato en enero de 2009 buscó fortalecer la democracia de su país a partir de incentivar la confianza de los ciudadanos de ese país. Para ello encontró en la apertura y transparencia del gobierno el mecanismo ideal. El segundo momento igualmente fue protagonizado por el propio Obama en septiembre de 2010 cuando al dirigirse a la Asamblea General de Naciones Unidas expuso nuevamente su propuesta. La consecuencia fue la conformación de la Alianza para el Gobierno Abierto, un organismo internacional que reunió a un grupo de países y organismos no gubernamentales para que establecieran estándares de desempeño y planes de acción para la implementación de políticas en la materia.

A decir de la OCDE el contexto económico incide al menos en tres grandes áreas: la eficiencia operacional del gobierno, la reducción en el costo de los errores y fraudes en beneficio de la administración pública, y el incremento de la recaudación fiscal (OECD, 2016). A estos factores debe sumarse uno particularmente importante: la posibilidad que tendrían los particulares y las empresas privadas de diseñar nuevos modelos de negocios a partir de la reutilización de los datos del gobierno por medio de plataformas o programación de aplicaciones (APIs).

Habiéndose definido el marco contextual del gobierno abierto, la propia Harrison refiere la existencia de tres actores principales: el gobierno, los innovadores representados por el sector tecnológico y los usuarios representados por los integrantes de la sociedad civil. Con el ánimo de identificar otros posibles actores en el modelo se consultaron los trabajos de autores como Dawes o Aguilera. Ambos coinciden en la presencia de tres actores, tal como se se puede observar en la Tabla I.

\begin{tabular}{lclc}
\hline & Actores & & Autores \\
\hline Gobiernos & Innovadores & Ciudadanos & $\begin{array}{c}\text { (Harrison T, } \\
\text { et al. 2012) }\end{array}$ \\
\hline $\begin{array}{l}\text { Productores } \\
\text { de datos }\end{array}$ & Innovadores & Usuarios & $\begin{array}{l}\text { (Dawes SS, } \\
\text { et al.,2016) }\end{array}$ \\
\hline $\begin{array}{l}\text { Administraciones } \\
\text { públicas }\end{array}$ & Compañías & Ciudadanos & $\begin{array}{l}\text { (Aguilera, U. } \\
\text { et al. 2017) }\end{array}$ \\
\hline
\end{tabular}

Tabla I. Actores del ecosistema del gobierno abierto

El común denominador de las perspectivas de los tres autores conduce a la ubicación del gobierno como un primer actor que se caracteriza por ser el 
generador o recolector de datos, en segundo lugar se encuentran a las tecnologías de información y comunicación, las cuales participan con la oferta de plataformas para la captura, almacenamiento, organización y difusión de los contenidos del gobierno. Finalmente, el ciudadano, entendido como el usuario final quien en conocimiento de las colecciones de datos es capaz de reutilizarlas para generar nueva información. Con ello sería capaz de participar objetiva y activamente en la toma de decisiones, ya sea por medio de foros de participación ciudadana, redes sociales o cualesquiera medios que provea el gobierno o que tenga a su alcance para hacer saber y valer su opinión.

Importante es en este punto recalcar la diferencia entre la información cualitativa y la cuantitativa que puede obtenerse por medio del modelo del gobierno abierto. La información cualitativa típicamente se obtendrá por medio de mecanismos vinculados al acceso a la información pública, mientras que la de carácter cuantitativa se obtendrá por la concentración de datos numéricos obtenidos de los registros que dan cuenta de los trámites de gobierno, de las contrataciones, de los censos, entre otros. Ahora bien, en este modelo de gobierno no solo promueve la continuidad del ejercicio del derecho de acceso a la información, sino que se va más allá al conducir al gobierno a que de manera proactiva libere el acceso a sus datos. De esta forma se confirma cómo el modelo del gobierno abierto se encuadra a los principios de movimiento open al permitir un libre acceso, uso y reutilización de los contenidos informacionales administrados por el gobierno. Esta idea se concreta en la práctica al colocar los datos del gobierno en formatos libres de derechos de propiedad intelectual, es decir, que para su manejo no se requiera la utilización de software propiedad de alguna persona o empresa que pueda solicitar compensaciones por el uso de sus soluciones. Para abundar en las características que deben contar los datos Ubaldi (2013) recopila las características que deben satisfacer los datos para ser útiles: estar completos, ser una fuente primaria, estar actualizados, ser accesibles, disponibles para su procesamiento computacional, no discriminatorios, no ser propiedad de alguien y no estar albergados en formatos o software propiedad de un privado o empresa.

Por su parte Dowes y sus coautores al proponer un esquema de valoración técnica infieren los requisitos mínimos que deben satisfacer los datos: a) formato, datos que se presentan en formatos libres de licencias, interoperables, y listos para usarse adecuadamente con herramientas de web semántica; b) metadatos, que exista una inequívoca correspondencia de los contenidos con sus campos de identificación; c) acceso, la permisibilidad de descarga de los data-sets en diferentes formatos para permitir una adecuada visualización de los contenidos; y d) calidad, que los contenidos se encuentren completos, libres de errores de captura y normalizados (Dowes et al., 2016).

En resumen, el corazón del modelo del gobierno abierto se encuentra en la recolección, organización y puesta a disposición de los datos que provienen de una variedad de fuentes. Tales datos al atender una serie de estándares permiten su reutilización y con ello la generación de nueva información. El fin último es el fortalecimiento de una democracia deliberativa que se apoye en un modelo estructurado para promover la participación ciudadana.

\subsection{Los intermediarios de información}

Gobierno, tecnología y usuarios se presentan en la literatura especializada como los actores principales del gobierno abierto, y a su vez, cada uno de ellos cuentan con su propio ecosistema compuesto por actores y necesidades. Por ejemplo, el gobierno debe lidiar con la encomienda de recolección y apertura de datos, la tecnología debe resolver la gestión voluminosa de los datos, y los usuarios tendrían que desarrollar la capacidad de interpretarlos. Para ninguna de las partes la tarea se perfila simple, y mucho menos para el modelo mismo de gobierno abierto que debe atender los intereses de todos los actores. A decir de Harrison y sus co-autoras, el verdadero valor del gobierno abierto no está en la gestión de los datos, sino en la interrelación de los actores. Es en ese lugar donde surgen la mayor cantidad de conflictos, pero también es el espacio donde hay mayor posibilidad de injerencia para incentivar (o modelar) el movimiento e interdependencia que debe existir entre los involucrados en el ecosistema (Harrison et al., 2012).

Los motivos de conflicto entre los actores son variados, pero el más recurrente proviene de las expectativas que existen sobre el desempeño de las tareas de cada una de las contrapartes. Ejemplo de ello puede darse con el ciudadano quien está acostumbrado al nivel de usabilidad que le ofrecen actualmente las redes sociales. Lo que mínimamente esperaría el usuario de los datos del gobierno es que se encuentren en una plataforma de fácil acceso por medio de dispositivos móviles o de escritorio, y una fácil interacción con los contenidos, ya sea para consultarlos o descargarlos, así como una disponibilidad en formatos libres que le permitan reutilizarlos para realizar combinaciones de datos e inclusive producir nuevas aplicaciones.

En el otro vértice del ecosistema se encuentra el gobierno, quien está obligado por leyes y compromisos políticos locales e internacionales a abrir datos que probablemente no tenga ordenados. A 
su vez, el propio gobierno desearía saber a qué tipo de información debería darle prioridad, considerando que de por sí la exigencia de apertura es alta, a ella debe sumarle la indicación que todo debe estar abierto. Por tanto, el gobierno requiere de la asistencia de la ciudadanía para priorizar la disponibilidad de datos que realmente aporten valor al modelo. Finalmente, el sector tecnológico que debe ocuparse en el posicionamiento de sus soluciones basadas en datos de gobierno.

Para empezar aliviar las tensiones existentes entre los actores, y poniendo por encima de ellas el interés por producir soluciones informacionales a favor del fortalecimiento de la participación ciudadana, se presenta la oportunidad de incluir a un agente que facilite e incentive el consumo de la información del gobierno. Esto ante la predecible realidad de la poca usabilidad de los datos por la complejidad que representa ubicarlos, descargarlos y darles un significado que anteceda una acción de participación ciudadana. Por este motivo, autores como Fast y Rinner (2917) retoman de la literatura especializada la presencia de agentes intermediarios, sean humanos o no humanos, que acorten la distancia entre los proveedores de datos abiertos y los usuarios finales a partir de ofrecer servicios, portales o plataformas.

El aporte que hace este tipo de agentes al modelo proviene principalmente de sus capacidades para realizar análisis estadísticos, para la visualización de datos y para el desarrollo de aplicaciones web. Estas son algunas de las habilidades que se asocian al concepto data literacy con el que se engloba una serie de conocimientos que buscan dar significado y utilidad a los conjuntos de datos obtenidos, en este caso, a partir de los contenidos provistos por el modelo del gobierno abierto. Estos agentes, al tener como materia prima de su actividad a los datos y como objetivo el hacerlos entendibles, han recibido el mote de infomediarios. El producto de su trabajo cada vez es más fácil encontrarlo en aplicaciones como las que se enlistan en la Tabla II, y son tantas que ha sido necesario clasificarlas. Johnson y Greene indican que entre los tipos de infomediarios de datos abiertos se encuentra los que provienen del propio gobierno, del sector privado, de las organizaciones no gubernamentales, de la academia y finalmente, de los medios (Johnson \& Greene, 2017).

\begin{tabular}{llll}
\hline Tipo & Autor & Nombre y descripción & URL \\
\hline Gobierno & $\begin{array}{l}\text { Instituto Nacional } \\
\text { de Estadística y } \\
\text { Geografía (México) }\end{array}$ & $\begin{array}{l}\text { DENUE, que permite ubicar la clasificación y } \\
\text { distancia geográfica entre unidades de negocio }\end{array}$ & $\begin{array}{l}\text { http://www.beta.inegi.org.mx/ } \\
\text { app/mapa/denue/ }\end{array}$ \\
\hline Sector privado & $\begin{array}{l}\text { Gobierno fácil } \\
\text { (México) }\end{array}$ & $\begin{array}{l}\text { Gobierno fácil, que ubica data-sets temáticos y } \\
\text { crea representaciones web para su fácil } \\
\text { comprensión }\end{array}$ & http://gobiernofacil.com/ \\
\hline $\begin{array}{l}\text { Organizaciones no } \\
\text { gubernamentales }\end{array}$ & Instituto Mexicano \\
para la Competitividad & $\begin{array}{l}\text { Índices del IMCO, que, por medio de } \\
\text { publicaciones y bases de datos permite } \\
\text { monitorear políticas públicas de competitividad y y } \\
\text { desarrollo económico en México }\end{array}$ & http://imco.org.mx/indices/\#!/ \\
\hline Academia & $\begin{array}{l}\text { Ricardo Haussmann, } \\
\text { Universidad de } \\
\text { Harvard }\end{array}$ & $\begin{array}{l}\text { Atlas de complejidad económica, que permite } \\
\text { identificar el nivel de participación de los } \\
\text { productos o servicios en la economía doméstica }\end{array}$ & http://atlas.cid.harvard.edu \\
\hline
\end{tabular}

Tabla II. Aplicaciones de gobierno abierto

\subsection{Las bibliotecas como infomediarios}

En palabras de Calzada y Marzal (2013) al público en general no le es fácil ubicar e interpretar los datos. Por tanto, tales usuarios potenciales requieren del apoyo de terceras personas o de agentes quienes puedan ofrecerles asistencia para la identificación e interpretación de tales colecciones de datos. En este punto es que las bibliotecas se perfilan como el agente natural de intermediación entre usuario y dato debido a su capacidad probada para gestionar colecciones y ofrecer servicios de información. Más aún, la posición de la biblioteca al interior de la sociedad es la de un actor neutral en cuyos espacios pueden convivir todos los integrantes de la localidad sin que medien intereses partidarios. Esta cualidad es de particular importancia en el proceso de la gestión de los servicios ofertados a partir de datos de gobierno, pues no existiría ningún interés por interpretar sus contenidos de manera tendenciosa. El proveer al interior de las bibliotecas servicios de información a partir de este tipo de datos es de particular importancia para abonar a los ideales de la democracia deliberativa. Este concepto tiene por fin último la conformación de una sociedad que se involucre activamente en la toma de decisiones públicas a partir de la deliberación con argumentos objetivamente documentados. Por tanto, la neutralidad de la biblioteca en el ofrecimiento de servicios de 
información basados en datos de gobierno abona a la conformación de un criterio colectivo sustentado en contenidos reales y actuales.

Para el bibliotecario profesional la gestión de datos abiertos se presenta como una oportunidad. Sus habilidades tradicionales de organización, preservación y difusión de materiales documentales se deben llevar a un nuevo nivel. Los datos requieren que tales habilidades se extiendan al ámbito de lo digital, seleccionando y preservando tales colecciones bajo principios de curaduría de datos. A su vez, la oferta de servicios de información se debe sustentar en nuevas habilidades de acceso, de medición crítica, interpretación, manipulación, abstracción, manejo, presentación y uso ético de los datos. El resultado esperado sería transformar los componentes tradicionales de la alfabetización informacional (information literacy) al data literacy tal como lo proponen los propios Calzada y Marzal (2013).

El resultado de tales transformaciones profesionales se empieza a evidenciar con algunos ejemplos. Es el caso de la Biblioteca Pública de Chattanooga quien no solo se encarga de ofrecer servicios de información basados en datos de gobierno, sino que se responsabiliza de la administración del portal de los datos de su gobierno. El resultado es la vinculación entre gobierno y usuarios que deriva en la utilización efectiva de los nuevos acervos, así como en la planeación de eventos alrededor de ellos como pueden ser hackatones o proyectos bajo el modelo makerspace. Otro ejemplo es la Biblioteca Pública de Boston que con el auspicio de la Knight Foundation desarrolló un proyecto de servicios de información basado en datos abiertos de gobierno y contrató a un datalibrarian que se encargue de ponerlo en marcha.

Como es visible, la gestión de los datos abiertos del gobierno al interior de las bibliotecas representa todo un proceso de revolución profesional. Los cambios presentes, más los que se avecinan no son eventos aislados, por el contrario. Ya se decía que el movimiento del gobierno abierto tiene entre sus antecedentes al movimiento open, y la tendencia de una data literacy igualmente encuentra su origen en los estándares del information literacy. Por tanto, la propuesta de incentivar a las bibliotecas como infomediarios del gobierno abierto se confirma como un paso natural y obligado en el proceso de avance de la profesión.

\section{Conclusiones}

Los profesionales de la información pueden encontrar una nueva área de desarrollo en el último eslabón del ciclo del gobierno abierto, es decir, en la entrega de los datos a la ciudadanía para su reutilización y generación de nueva información. Igualmente se hace evidente que desde el proceso mismo de organización de los datos al interior de las dependencias de los gobiernos es necesaria su participación. En la medida que el bibliotecario se involucre en cada una de las etapas que componen el ecosistema del gobierno abierto, coadyuvará a la concreción del modelo que aspira a la conformación de una ciudadanía objetivamente informada y participativa en los procesos de deliberación pública.

\section{Referencias}

Aguilar-Villanueva, Luis. (2017) Gobernanza: el nuevo modelo de gobernar. Conferencia, Biblioteca Palafoxiana. Junio 07. Puebla-México.

Aguilera, Unai; Peña, Oscar; Belmonte, Oscar; López-de-Ipiña, Diego (2017). Citizen-centric data services for smarter cities. // Future generation computer system. 76, 234-247.

Calzada-Prado, J.; Marzal, M. A. (2013) Incorporating data literacy into information literacy programs: core competencies and contents. // Libri: International Journal of Libraries \& Information Services. 63:2, 123-134.

Dawes, S. S., Vidiasova, L.; Parkhimovich, O. (2016). Planning and designing open government data programs: An ecosystem approach. // Government Information Quarterly. 33:1, 15-27.

Fast, Victoria; Rinner, Claus (2017). Mediating open data: providers, portals and platforms. // URISA Journal. 28, 7-8.

García-Sánchez, María Isabel (2007). La nueva gestión pública: evolución y tendencias. // Presupuesto y Gasto Público. 47:1, 37-64

Gasco, Mila. (2014) Avances y desafíos del gobierno abierto en Iberoamérica. //Conferencia virtual internacional. https://www.youtube.com/watch?v=5J0-Y42bWhY (Marzo 30, 2018)

Harrison, Teresa; Pardo, Theresa; Cook, Meghan (2012) Creating open government ecosystems: a research and development agenda. // Future Internet. 4. 900-928.

Johnson, A.; Green, S. (2017). Who are government open data infomediaries? A preliminary scan and classification of open data users and products. // URISA Journal. 28, 9-18.

Naser, A.; Ramírez, A. (2014). Plan de gobierno abierto, una hoja de ruta para los gobiernos de la región.

OECD (2016). Open government data review of Mexico, data reuse for public sector impact and innovation. // OECD Digital Government Studies. Paris : OECD Publishing.

OECD (2017). Government at a glance. Chapter 10. Open Government. Paris: OECD Publishing. 183-193.

Pierre, J. (2000). Debating Governance: authority, steering and democracy. Oxford University Press.

Ubaldi, B. (2013) Open government data: towards empirical analysis of open government data intiatives. // OECD working papers on public governance. 22.

Enviado: 2018-4-01. Segunda versión: 2018-10-02. Aceptado: 2019-06-10. 